Revista de Iniciação Científica em Odontologia. 2018;16(3):101-108

ISSN 1677-3527 - DOI: 10.4034/RevIC0.2018.16.3.10

Relato de Caso

\title{
Transposição Dentária Mandibular com Diagnóstico Precoce: Relato de Caso
}

\author{
Mandibular Dental Transposition with Early Diagnosis: Case Report
}

\author{
Marília Michele Paixão de Oliveira1; Rejane Targino Soares Beltrão²
}

${ }^{1}$ Aluna do Programa de Pós-Graduação em Odontologia da Universidade Federal da Paraíba UFPB, João Pessoa - Paraíba.

2Professora Adjunto de Ortodontia e Clínica Infantil do curso de Odontologia da UFPB, João Pessoa/PB, Brasil.

Autor para correspondência:

Marília Michele Paixão de Oliveira. Rua Abelardo do Rego, $n^{\circ}$ 127. Mangabeira VIII, João Pessoa, Paraíba. CEP: 58059-352. E-mail: mari-michele1@hotmail.com.

\begin{abstract}
Resumo
Introdução: transposição dentária é uma anomalia relacionada à erupção ectópica ou à falha na erupção dentária, que pode afetar o desenvolvimento físico, psíquico e social da criança, e que pode ser de difícil resolução clínica. O objetivo deste artigo é apresentar um estudo de caso clínico sobre transposição dentária, com uma alternativa de tratamento ortodôntico. As informações foram obtidas através da coleta e análise de dados contidos em prontuário odontológico, coligado ao exame clínico do paciente, após consentimento do responsável. Relato de Caso: Paciente leucoderma, oito anos de idade com diagnóstico de transposição dentária entre o canino e o incisivo lateral inferior esquerdo. Foi realizado tratamento ortodôntico conservador, envolvendo a correção ortodôntica dos dentes transpostos. Conclusão: O caso apresentado cumpriu com o esperado, de forma que a transposição dentária parcial entre o dente 33 e 32 foi totalmente corrigida, sem danos aos tecidos de suporte e evitando possíveis perdas dentárias, além da correção da má oclusão, estabelecendo-se todas as excursões mandibulares normais, uma boa estética do sorriso e a normalidade das funções estomatognáticas.
\end{abstract}

Descritores: Transposição. Erupção dentária. Ortodontia. 


\begin{abstract}
Introduction: Dental transposition is an anomaly related to ectopic eruption or failure of dental eruption, which can affect the physical, psychological and social development of the child, and may be difficult to be clinically solved. The aim of this article is to present a clinical case study on dental transposition with an alternative orthodontic treatment. The information was obtained through the collection and analysis of data contained in dental records, related to the clinical examination of the patient, with the consent of the person responsible Case Report: Leucoderma patient, eight years old with diagnosis of dental transposition between the canine and the left lateral lateral incisor. Conservative orthodontic treatment was performed, involving the orthodontic correction of the transposed teeth. Conclusion: The presented case complied with the expected, the partial dental transposition was totally corrected, without damages to the supporting tissues and avoiding possible dental losses, besides the correction of the malocclusion, establishing all the normal mandibular excursions, a good aesthetic of the smile and the normality of the stomatognathic functions.
\end{abstract}

Keywords: Impacted tooth. Ectopic tooth eruption. Orthodontics.

\title{
Introdução
}

A transposição dentária é considerada uma anomalia na erupção dentária, na qual dois dentes adjacentes ou não, localizados no mesmo quadrante do arco dentário se desenvolvem e irrompem em posições invertidas, ocorrendo uma modificação na sequência normal de erupção'.

A etiologia da transposição dentária apesar de não estar completamente definida, é considerada como sendo multifatorial. Evidências sugerem que o aparecimento dessa anomalia, esteja relacionado à perda precoce ou retenção de dente decíduo, deslocamento do germe dentário durante a odontogênese, inadequado comprimento do arco, volume dentário excessivo ou trauma de dente decíduo, fatores genéticos com causas multifatoriais de herança, assim como doenças ósseas ou fatores locais, cistos e tumores². A maior prevalência dos casos é do sexo feminino, com maior ocorrência na maxila do que na mandíbula ${ }^{3}$. Algumas anomalias dentárias podem ser observadas associadas à transposição, como incisivos laterais superiores ausentes ou conóides; giroversões; microdontia; macrodontia, impactações, retenções de dentes decíduos, agenesias dentárias e hipoplasia de esmalte ${ }^{4}$.

A transposição classifica-se em completa e parcial de acordo com o posicionamento da coroa, raízes e ápices dos elementos dentários envolvidos. É considerada completa quando o dente encontra-se em posição completamente trocada no arco dentário, existindo um paralelismo entre suas raízes e a dos demais dentes envolvidos, e parcial quando os ápices radiculares permanecem em suas posições originais, e somente as coroas sofrem transposição ${ }^{5}$. 
O diagnóstico precoce dessa anomalia é um fator preponderante para o prognóstico e solução dos casos. Quando o problema é diagnosticado na infância ou adolescência e o tratamento é iniciado rapidamente, pode evitar complicações como, por exemplo, a reabsorção radicular do elemento que está irrompido, que é causada pelo elemento dentário transposto e a perda do elemento dentário adjacente ${ }^{6,7}$. Assim como em outras anomalias, o diagnóstico precoce pode trazer menos ônus financeiro, emocional e dor ao paciente'1.

Diante do exposto, visto que existem poucos relatos na literatura sobre essa anomalia dentária, o objetivo deste artigo é apresentar um estudo de caso clínico de uma transposição dentária entre o canino inferior esquerdo (33) e o incisivo lateral inferior esquerdo (32).

\section{Relato de caso}

As informações foram obtidas através da coleta e análise de dados contidos em prontuário odontológico, coligado ao exame do sujeito da pesquisa, após consentimento do responsável.

A paciente, sexo feminino, 8 anos de idade, procurou a Clínica Integrada Infantil da Universidade Federal da Paraíba, para tratamento ortodôntico, visando a correção de más oclusões. Ao exame clínico inicial observou-se que a paciente se encontrava na dentadura mista e apresentava retenção dos elementos decíduos (72) e (73), transposição dentária do canino inferior esquerdo (33) e incisivo lateral inferior esquerdo (32) (Figura 1), sem outras anomalias associadas, que foi posteriormente confirmada pela radiografia panorâmica (Figura 2).

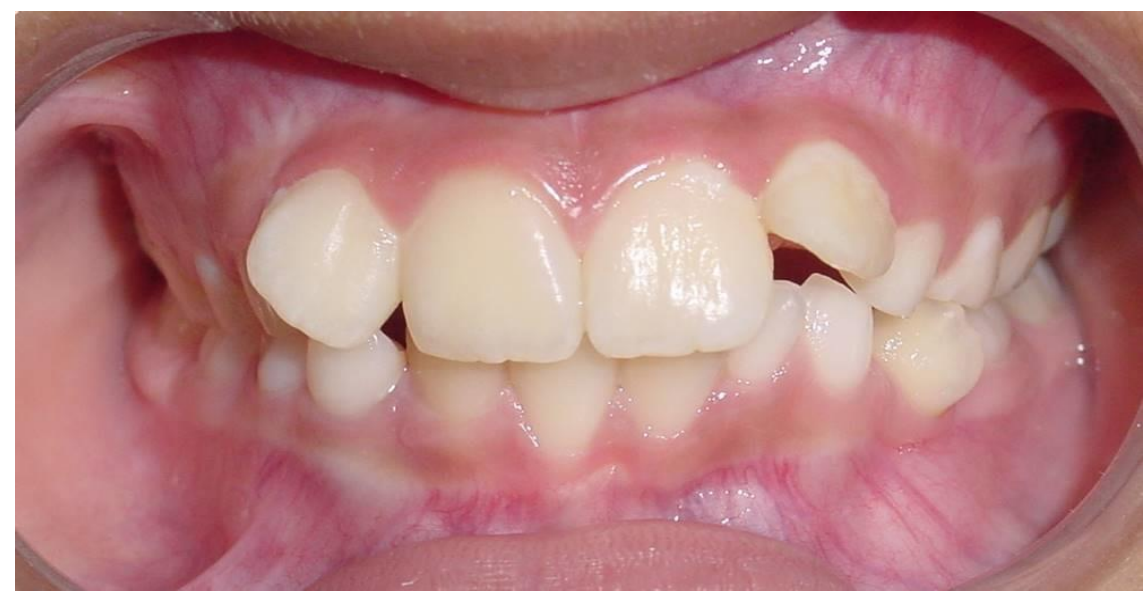

Figura 1. Fotografia intrabucal vista frontal. 


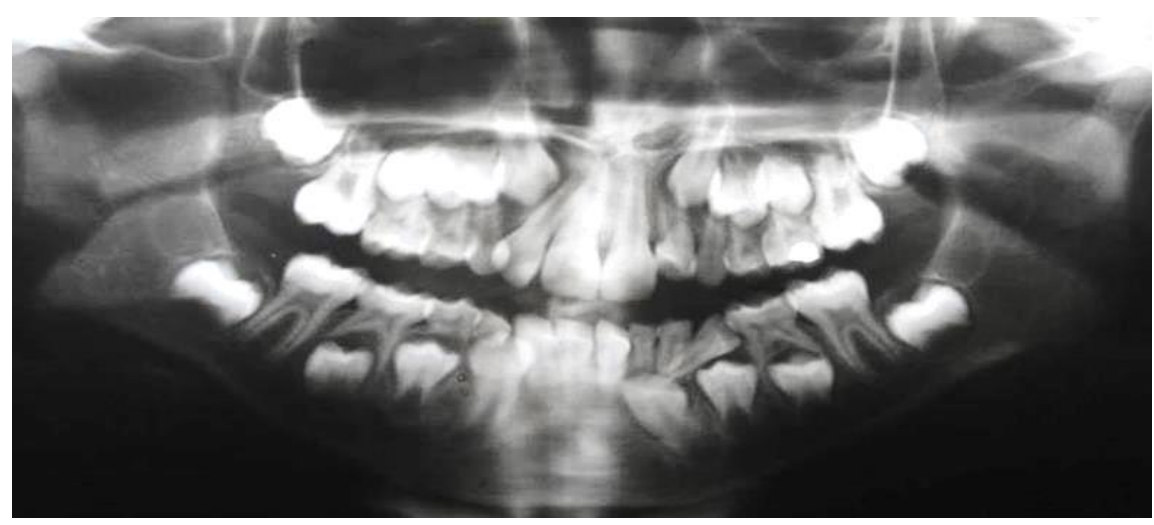

Figura 2. Radiografia panorâmica com transposição dentária parcial dos elementos 33 e 32 .

O tratamento ortodôntico teve como objetivo tratar a transposição dentária e demais problemas de oclusão, mantendo a estética favorável da paciente. O plano de tratamento proposto correspondeu à extração dos elementos (72) e (73), instalação de um aparelho ortodôntico fixo (nivelamento 4x2), mecânica por meio de elásticos para tracionar e mesializar o incisivo lateral (32) e (33). Utilizou-se como ancoragem sido o arco lingual de Nance inferior, elásticos correntes inseridos no elemento (33) e elemento (32). Iniciou-se a mecânica ortodôntica sempre com forças leves, possibilitando assim a transposição por mesialização do incisivo lateral inferior esquerdo (32) (Figura 3).

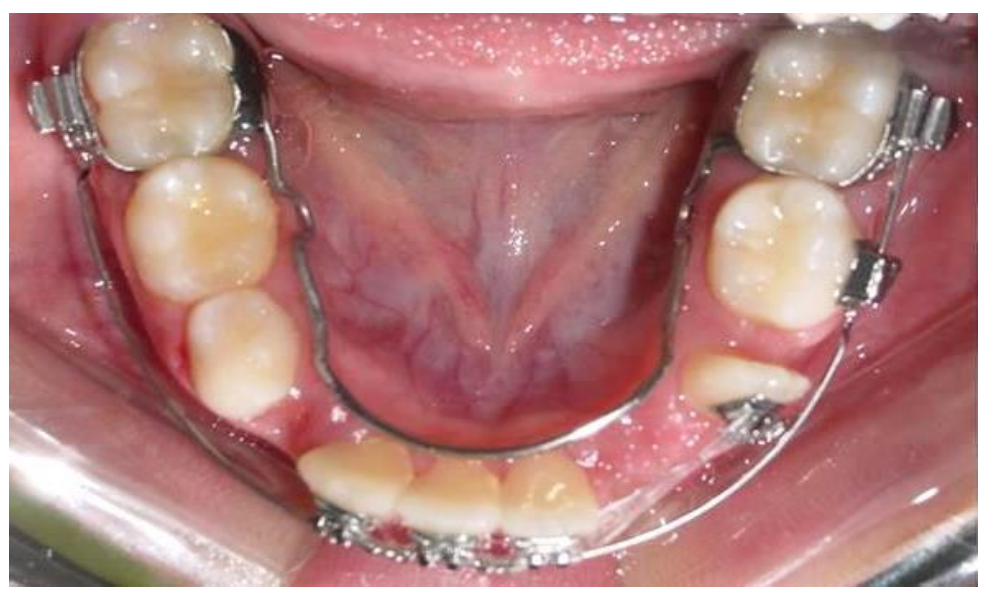

Figura 3. Fotografia intrabucal vista frontal com instalação de aparelho fixo no arco inferior e tracionamento do elemento 32.

Ao final do tratamento o aparelho fixo foi removido e foi instalada uma contenção fixa com fio de aço inoxidável aderida a lingual superfícies dos elementos (33) a (23), com resina 
fotopolimerizável e solicitada a radiografia panorâmica mostrando o final do tratamento (Figuras 4, 5 e 6$)$.

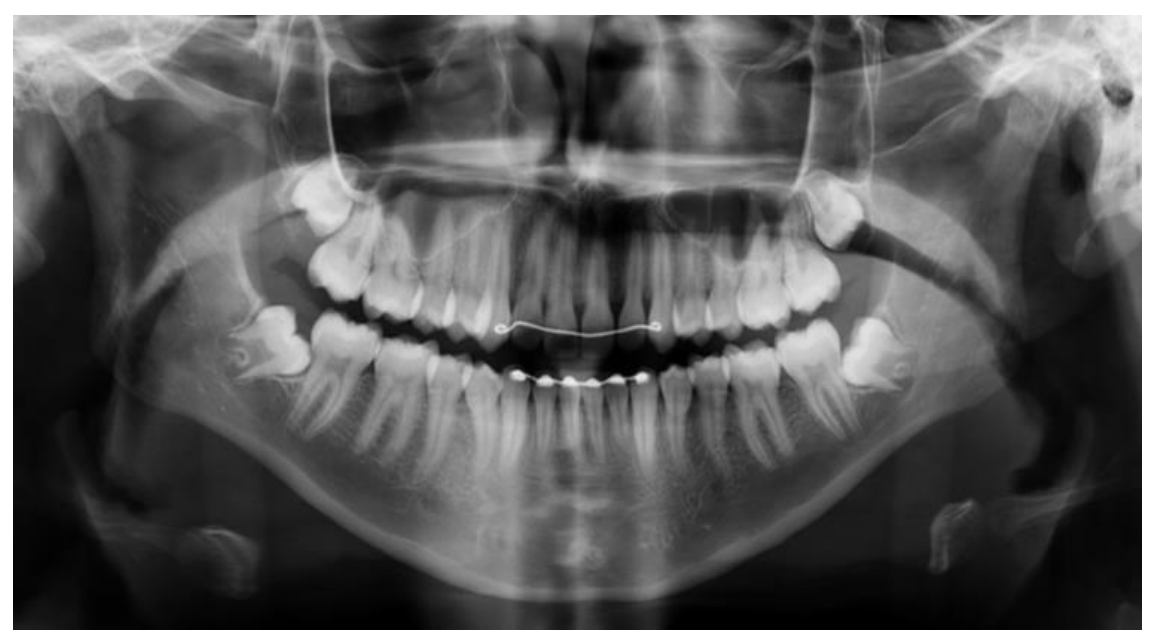

Figura 4. Radiografia panorâmica mostrando o tratamento finalizado.

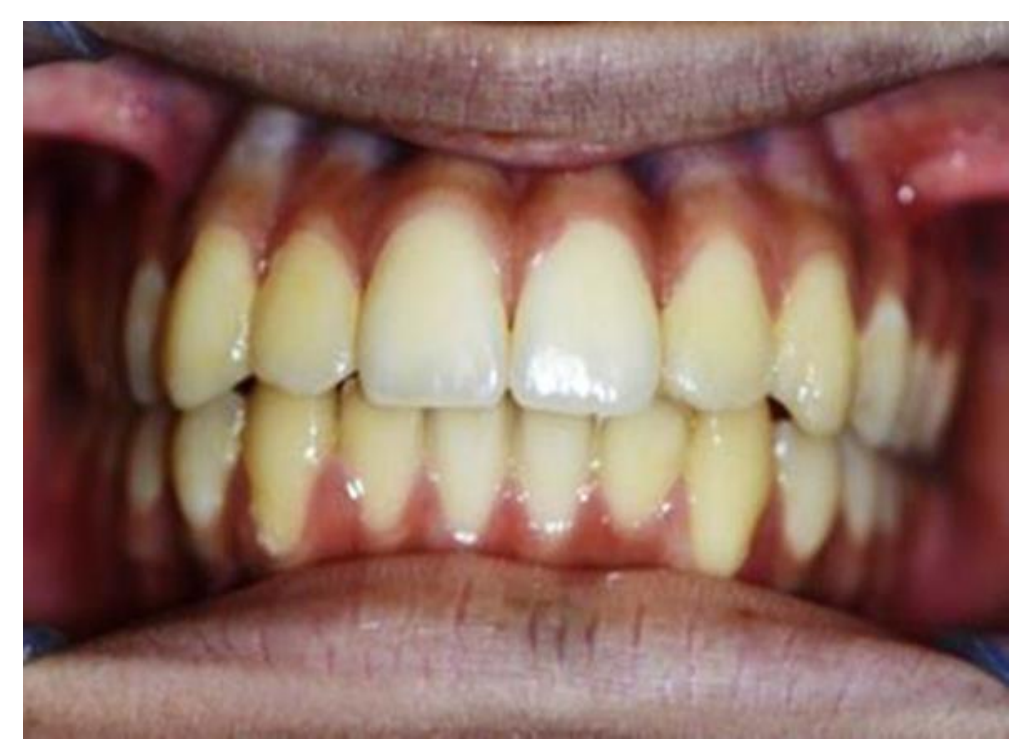

Figura 5. Fotografia intrabucal vista frontal caso finalizado. 


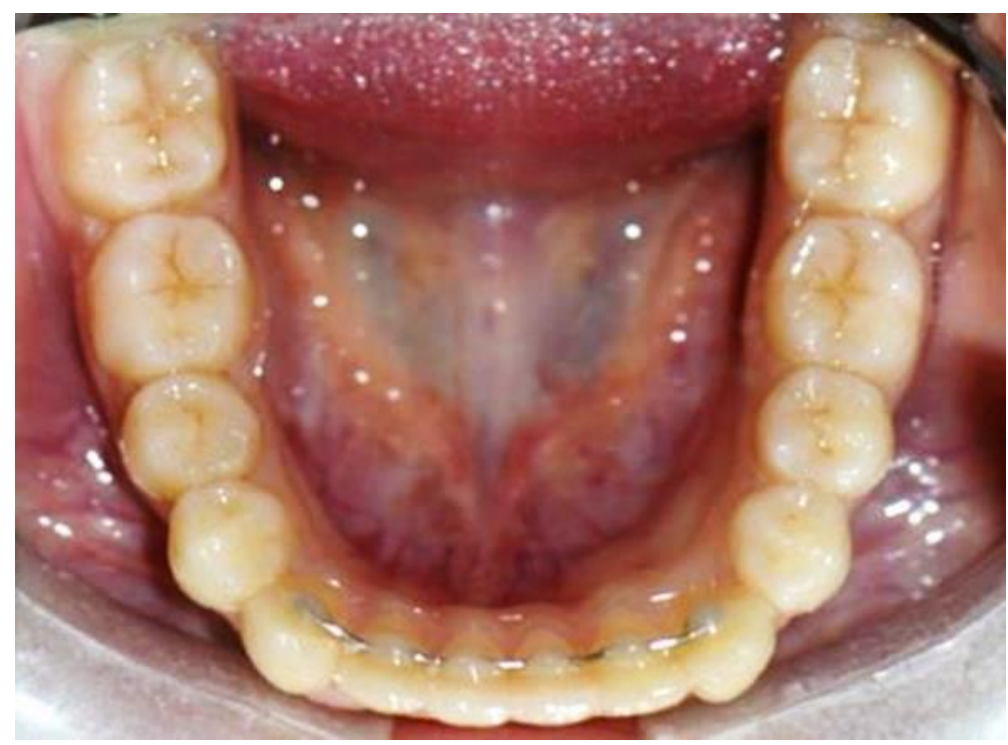

Figura 6. Fotografia oclusal do arco inferior com contenção fixa caso finalizado.

\section{Discussão}

A transposição dentária não apresenta uma única etiologia na literatura, sendo atribuída a vários fatores. Em estudos afirmaram que evidências sugerem que o aparecimento dessa anomalia estaria relacionado à perda precoce ou retenção de dentes decíduos, inadequado comprimento do arco, volume dentário excessivo ou trauma de dente decíduo $0^{6,8}$. Considerando-se as características clínicas e radiográficas do presente caso clínico, a hipótese etiológica seria a retenção de dentes decíduos, estando dentro dos fatores mencionados na literatura.

A transposição unilateral é mais comum do que transposição bilateral e o lado esquerdo é o lado mais prevalente ${ }^{3,7-10}$. O que está de acordo com o caso clínico aqui apresentado. Considerando-se os dentes envolvidos, a transposição ainda pode acontecer em: canino no local do primeiro pré-molar; incisivo lateral no local do canino; canino no local do primeiro molar; incisivo central no local do incisivo lateral; o canino no local do incisivo central ${ }^{3,7,8,10,11}$. Nos estudos citados anteriormente, observa-se que o canino é o elemento mais envolvido em processos de transposição dentária bem como demonstrado por esse caso clínico, em relação a localização a ocorrência transposição é maior na maxila e no presente caso ocorreu na mandíbula sendo menos comum. 
No caso apresentado, optou-se por uma mecânica por meio de elásticos para tracionar e mesializar o incisivo lateral (32), pois esta abordagem seria menos invasiva, uma vez que o canino (33) ainda se encontrava incluso. Estudos também foi utilizaram essa técnica como forma de intervenção, tendo como benefícios a manutenção de uma estética favorável, pois o caso pode ser finalizado com os dentes em sua posição habitual e não transposta, sem a necessidade de terapêuticas adicionais como modificação estética com resina composta ${ }^{12}$.

Neste clinico não houve dificuldades durante o tratamento, mesmo a paciente sendo uma criança, foi colaborativa e possuía uma boa higiene oral. A idade foi um aspecto positivo, pois devido à precocidade do diagnóstico do caso aqui apresentado foi possível a utilização de uma mecânica ortodôntica em que a intervenção ocorresse de forma minimamente invasiva, preservando-se os tecidos dentários, com controle da relação das raízes. Esse resultado também foi encontrado em outros estudos onde o diagnóstico e tratamento foi realizado precocemente ${ }^{4,12}$.

Em relação ao melhor período para realizar o tratamento, a literatura relata que o diagnóstico da transposição dentária deve ser realizado de forma precoce para favorecer 0 prognóstico da correção ortodôntica ${ }^{4,6,7}$. Podendo ser feito quando os dentes envolvidos estão irrompidos, ou quando apenas um é irrompido e o outro aparece transposto de maneira intraóssea ${ }^{8}$.

\section{Conclusão}

De acordo com as considerações clínicas e a literatura exposta, o caso apresentado cumpriu com o esperado, de forma que a transposição dentária parcial entre o dente 33 e 32 foi totalmente corrigida, sem danos aos tecidos de suporte e evitando possíveis perdas dentárias, além da correção da má oclusão, estabelecendo-se todas as excursões mandibulares normais, uma boa estética do sorriso e a normalidade das funções estomatognáticas. É importante ressaltar que o sucesso do tratamento desse caso se deu pelo diagnóstico precoce da transposição dentária, enquanto parcial, o que favoreceu o tratamento.

\section{Referências}

1. Gebert T, Palma V, Borges A, Volpato L. Dental transposition of canine and lateral incisor and impacted central incisor treatment: A case report. Dental Press J Orthod; 2014; 19(1):106-112. 
2. Ramos D, Daruge Júnior E, Daruge E, Antunes F, Heléndez B; Francesquini-Júnior L. Transposición dental y sus implicaciones éticas y legales. Rev ADM. 2005;62(5):185-190.

3. Gomberg $\mathrm{E}$ et al. Transposição dentária maxilar bilateral: relato de um caso Revista de Ciências Médicas e Biológicas. 2010; 9(3):263-265.

4. Shapira Y, Tamar F, Kadry R, Schonberger S, Shpack N. Mandibular Symmetrical Bilateral Canine-Lateral Incisors Transposition: Its Early Diagnosis and Treatment Considerations. Case Reports in Dentistry. 2016;1(2):1-6.

5. Locks A, Sória M, Costa C; Ribeiro G; Rocha R. A importância da correção precoce da transposição dental na mandíbula: apresentação de caso clínico. J Bras Ortodon Ortop Facial. $2001 ; 6(34): 338-344$.

6. Capelozza Filho L, Cardoso MA. Tratamento de transposição de canino e pré-molar superior unilateral abordagem por meio de mecânica segmentada. Rev. Clin. Ortodon. Dental Press. 2007; 6(3):73-85.

7. Borba DP et al. Irrupção ectópica de incisivo lateral inferior-relato de caso. RFO. 2008; 13(2):78-82.

8. Costa LED et al. Transposição dentária: estudo de prevalência em escolares na cidade de João Pessoa-PB. Pesquisa brasileira em odontopediatria e clínica integrada. 2010; 10(1):107-112.

9.Babacan $\mathrm{H}$, kilic $\mathrm{B}$, Bicakci A. Maxillary canine-first premolar transposition in the permanent dentition. Angle Orthod. 2008; 78(5):954-960.

10. Praxedes OJ, Caldas S; Medeiros AM. Transposição dentária: um desafio na clínica ortodôntica - relato de caso. Rev Clin Ortodon Dental Press. 2006; 4(2):75-84.

11. Synodinos PN, Polyzois I. Maxillary canine-first premolar transposition in the permanent dentition: treatment considerations and a case report. J Ir Dent Assoc. 2011; 56(6):264-267.

12. Mendes $P$, Valarelli F, Freitas $K$, Cançado R, Vitor L, Oliveira T. Diagnóstico da transposição dentária na ótica da clinica ortodôntica: utilização de tomografia computadorizada com feixe cônico. Revista UNINGÁ. 2013; 36(1):101-113. 